MAGNETOHYDRODYNAMICS Vol. 54 (2018), No. 1-2, pp. 115-120

DOI: $10.22364 / \mathrm{mhd} .54 .1-2.20$

\title{
AZIMUTHAL DEPENDENCE OF THE ACOUSTOMAGNETIC EFFECT IN MAGNETIC FLUIDS
}

\author{
I.A. Shabanova ${ }^{1}$, A.M.Storozhenko ${ }^{1}$, A.O. Tantsyura ${ }^{1}$, I.M. Aref'ev ${ }^{2}$ \\ 1 Southwest State University, 94, 50 let Oktyabrya str., 305040 Kursk, Russia \\ ${ }^{2}$ Ivanovo Power Engineering University, 34 Rabfakovskaya str., 153003 Ivanovo, Russia
}

The paper describes the results on measuring the dependence of the acoustomagnetic effect on the angle between the magnetic field and the measuring inductive coil. We measured the sound speed in a magnetic fluid-glass tube system for two samples of nanodispersed magnetic fluid. The samples are disperse systems of single-domain magnetite particles in a hydrocarbon (kerosene, mineral oil) medium stabilized by oleic acid. The results are in good agreement with the previously published theory. One can use our experimental data to calculate physical (magnetic and geometric) parameters of magnetic nanoparticles applying acoustogranulometric technique.

Introduction. Magnetic fluids are colloidal fluids consisting of nanoscale ferromagnetic particles suspended in a carrier fluid. All nanoparticles are thoroughly coated with a surfactant to prevent agglomeration because of the surfactant's Van der Waals force [1]. The first magnetic fluid was synthesized in 1963 and since that time the magnetic, rheological, acoustic and other properties of such dispersed systems have been studied. Due to the fact that the magnetic fluids become strongly magnetized in the presence of a comparatively weak magnetic field, they have various applications, for example, in mechanical engineering, electronic devices, materials science research, loudspeakers and so on [2].

The intensive Brownian motion of magnetic nanoparticles results in high macroscopic homogeneity of magnetic fluids. In a magnetic field, the magnetic moments of ferromagnetic nanoparticles begin to align with the field. The more is magnetic field strength, the more ordered is the dispersed system. Under a strong magnetic field $(\sim 1 \mathrm{~T})$, magnetic fluids reach their saturation magnetization when almost all nanoparticles follow in the field direction [3].

When a sound wave spreads inside the magnetic fluid, a perturbation of its magnetization occurs. If one locates an inductive coil near the column of magnetic fluid, an e.m.f. will be induced in the coil (Faraday's law of induction). Such phenomenon in magnetic fluids was called acoustomagnetic and it was described in details by Polunin [4-5]. The acoustomagnetic effect allows detecting acoustic oscillations and studying the acoustic field inside magnetic fluids [6-7]. The amplitude of the e.m.f. strongly depends on the angle between the magnetic field and the measuring inductive coil. We investigated the values of the e.m.f. during the rotation of a permanent magnet which created a magnetic field. The data on the sound speed in the magnetic fluid-glass tube system for two samples of nanodispersed magnetic fluids are also presented.

1. Existing theory. Let us consider a situation, when a cylindrical cell of diameter $d$ is filled with a magnetic fluid and exposed to a uniform magnetic field directed perpendicularly to it. An inductive coil is placed, as shown in Fig. 1a, and has the parameters $L \times h \times d$ (length $\times$ height $\times$ diameter). The coil is placed close to the tube surface and can rotate easily (Fig. $1 b$ ).

The angle between the magnetic field and the normal of the coil is $\varphi$. We are interested in how the amplitude of the e.m.f. in the coil due to the acoustomagnetic 


\section{I.A. Shabanova, A.M. Storozhenko, A.O. Tantsyura, I.M. Aref'ev}

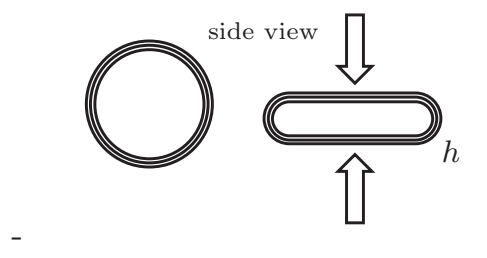

(a)

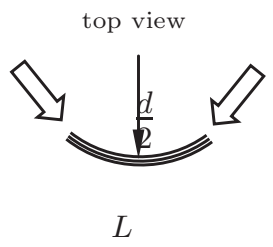

$L$

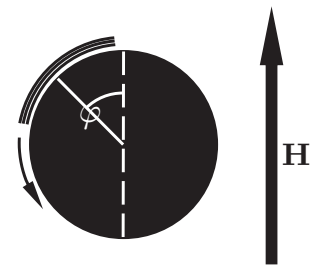

(b)

Fig. 1. Schematic presentation of the problem.

effect changed when $\varphi$ changes from 0 up to $2 \pi$. Using the model [8], this problem has been theoretically solved in [7], so in this paper we quote only the resulting formula. The e.m.f. amplitude is

$$
e=-\mu_{0}(1-N) N_{\mathrm{B}} h d \delta \dot{M} \sin (L / d) \cos \varphi,
$$

where $\mu_{0}$ is the magnetic constant, $N=0.5$ is the demagnetizing factor $[9,10]$, $N_{\mathrm{B}}=300$ is the number of turns in the coil, $\delta \dot{M}$ is the equilibrium value of the increment of magnetization per time. Hence, the value of the e.m.f. induced in the coil is proportional to $\cos \varphi$.

2. Experimental setup. In our acoustic experiments, we apply the idea of ultrasound interferometer [7]. The experimental setup is schematically shown in Fig. 2. The signal from a sound oscillation generator 1 comes to an oscilloscope 2 and to a piezo plate 3 arranged in the acoustic cell 4. The sound signal forms a standing wave when propagating through the magnetic fluid confined in a glass tube 5. The variable e.m.f. from the inductance coil 6 closely adjoining the tube comes to the oscilloscope 2. The measuring inductive coil is rigidly fixed on the cinematic unit of a cathetometer (not shown). The magnetic field is produced by a strong permanent magnet \%. A magnetic fluid fills the glass tube, the bottom of which is located between the poles of the permanent magnet. The height of the magnetic fluid column is $200 \mathrm{~mm}$.

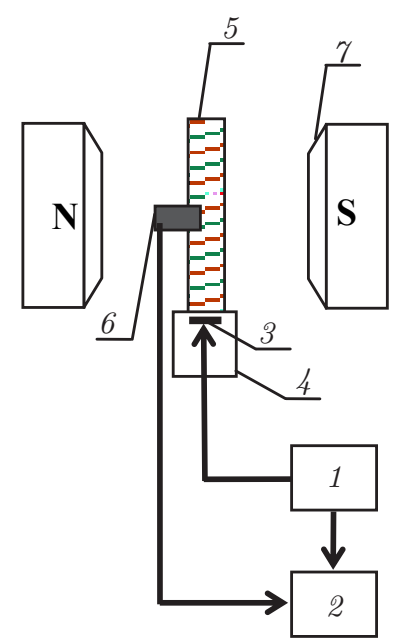

Fig. 2. Schematic presentation of the experimental setup. 


\section{Azimuthal dependence of the acoustomagnetic effect in magnetic fluids}

The parameters of the glass tube are the following: the Young's modulus $E=7.26 \cdot 10^{10} \mathrm{~Pa}$, the Poisson ratio 0.21 , the density $\rho_{\mathrm{t}}=2400 \mathrm{~kg} / \mathrm{m}^{3}$, the longitudinal wave velocity $c_{\mathrm{p}}=5500 \mathrm{~m} / \mathrm{s}$; the outer and inner radii, respectively, $R_{1}=8 \mathrm{~mm}, R_{2}=6.9 \mathrm{~mm}$, the wall thickness $b=1.1 \mathrm{~mm}$, the tube length $200 \mathrm{~mm}$.

The experiment was performed taking into account the optimal conditions, i.e. noise proof, control of the temperature $T=31 \pm 0.2^{\circ} \mathrm{C}$ and the constant voltage on the piezoelectric element $U=40 \pm 0.5 \mathrm{~V}$. The acoustic cell with the magnetic fluid was set in the pole gap in such way that the magnetic field lines were perpendicular to the tube axis.

The sensor moves along the tube with an accuracy of $0.01 \mathrm{~mm}$ due to the cathetometer used. Its distance is the central area of the pole gap and the adjacent areas of the non-uniform field.

First, we choose a frequency of the ultrasound oscillations at which an apparent standing wave occurs in the magnetic fluid column. The right choice could be verified in a simple way. When moving slowly the inductive coil upward and downward, maxima and minima of the signal appear periodically on the screen of the oscilloscope. They correspond to the nodes and anti-nodes of the standing wave. After amplification and analog-to-digital conversion, the signal from the inductive coil was processed by a PC using the LabView software, providing the experimental results.

When the coordinates of the nodes and anti-nodes of the standing wave are determined, it is easy to calculate the sound wavelength and then the sound speed as a product of frequency and wavelength. According to our estimations, the error of the sound speed value in the magnetic fluid filling the tube did not exceed $5 \%$ [11].

3. Samples. The study was carried out using samples of the magnetic fluids synthesized at the Scientific Research Laboratory of Applied Ferrohydrodynamics (ISPU, Ivanovo, Russia) by the method of chemical condensation [12]. The samples consist of single-domain spherical nanoparticles of magnetite $\mathrm{Fe}_{3} \mathrm{O}_{4}$ stabilized by oleic acid $\mathrm{C}_{8} \mathrm{H}_{17} \mathrm{CH}=\mathrm{CH}\left(\mathrm{CH}_{2}\right)_{7} \mathrm{CO}-\mathrm{O}^{-} \mathrm{H}^{+}$and dispersed in a hydrocarbon medium (kerosene, mineral oil). The physical characteristics of the samples are listed in Table 1. The magnetization curves of the magnetic fluids are close to each other and shown in Fig. 3. To obtain the magnetization curves, we used a ballistic technique [1].

Table 1. Physical characteristics of the samples.

\begin{tabular}{rcc}
\hline \hline & MF1 & MF2 \\
\hline Medium & mineral hydrocarbon oil & kerosene \\
Density, $\left[\mathrm{kg} / \mathrm{m}^{3}\right]$ & 2036 & 2075 \\
Concentration of solid state, $[\%]$ & 29.7 & 31.4 \\
Saturation magnetization $[\mathrm{mT}]$ & 38 & 39 \\
Average diameter of a particle, $[\mathrm{nm}]$ & 10 & 10 \\
\hline \hline
\end{tabular}

Table 2. Sound speed measurement results.

\begin{tabular}{ccccc}
\hline \hline Sample & $\begin{array}{c}\text { Frequency } \\
\text { range, }[\mathrm{Hz}]\end{array}$ & $\begin{array}{c}\text { Mean value of } \\
\text { sound speed, [m/s] }]\end{array}$ & $\begin{array}{c}\text { Absolute error } \\
\text { of sound speed }\end{array}$ & $\begin{array}{c}\text { Relative error } \\
\text { of sound speed }\end{array}$ \\
\hline MF1 & $46 \ldots 66$ & 1026 & 38 & $3.7 \%$ \\
MF2 & $36 \ldots 64$ & 952 & 85 & $4.9 \%$ \\
\hline \hline
\end{tabular}


I.A. Shabanova, A.M. Storozhenko, A.O. Tantsyura, I.M. Aref'ev

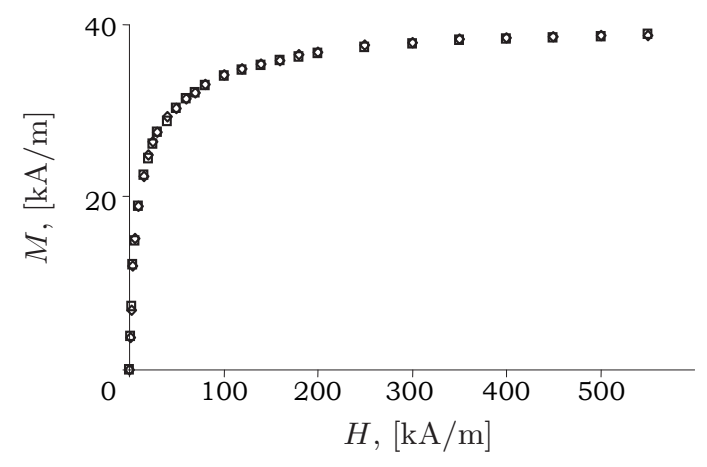

Fig. 3. Magnetization curves of the studied magnetic fluid samples.

This type of magnetic fluids is the most widespread. They are highly homogeneous and stable for a long time. The important advantages of these fluids are also the simple synthesis and the low cost of precursors. Therefore, such colloids are often chosen as research objects and are widely used in different applications.

4. Results and discussion. Using the described experimental setup, we measured the sound speed in the magnetic fluid-glass tube system for samples MF1 and MF2 at different frequencies. The results are illustrated in Table 2. The dispersion of values is in the range of the mentioned measurement error.

There is some doubt that the presence of a steady magnetic field could lead to a distortion of the data due to the dependence of the sound speed in MF on the magnetic field strength. Such uncertainty is unjustified, because in [13] it has been proved that within the measurement error the sound speed in a stable magnetic fluid does not depend on the magnetic field strength up to $\sim 500 \mathrm{kA} / \mathrm{m}$.

To obtain the azimuthal dependence of the acoustomagnetic effect in magnetic fluids, we carried out an experiment when the conductive coil was fixed and the permanent magnet rotated coaxially with the tube filled with the MF. The e.m.f. dependence in the coil on the angle $\varphi$ between the normal line $\mathbf{n}$ of the coil and the magnetic field direction $\mathbf{H}$ is rather specific. One can see it in Fig. 4, where the experimental values of the e.m.f. are presented in relative units $\varepsilon / \varepsilon_{\max }$. Here,

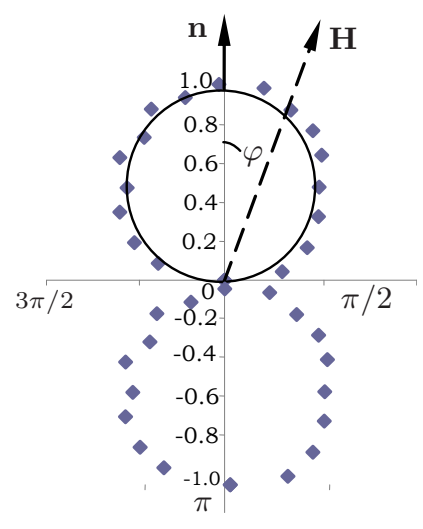

Fig. 4. The e.m.f. dependence in the coil on the angle $\varphi$ between the normal line $\mathbf{n}$ of the coil and the magnetic field direction $\mathbf{H}$. 
Azimuthal dependence of the acoustomagnetic effect in magnetic fluids

the distance between the zero-point and the experimental point is the relative amplitude of the e.m.f.

These experimental results are in good agreement with the existing theory described in details in [7]. Referring to the obtained experimental results, we can suggest to place the measuring inductive coil in such a way that its symmetry axis is parallel to the magnetic field.

The presented experimental data can be further used for calculating the physical (magnetic and geometric) parameters of magnetic nanoparticles in a magnetic fluid by the acoustogranulometric method [14] or in other acoustic investigations [15-16].

On balance, this interferometric methodology for determining the sound speed in a magnetic fluid has very good prospects due to its simple realization, that is, the experimental setup and the measurement technique. The technique utilizes the acoustomagnetic effect which can be observed in a magnetized magnetic fluid when elastic oscillations occur inside.

We calculated the speed of the ultrasonic wave propagation in the system magnetic fluid-glass tube for two samples of nanodispersed magnetic fluids, which belong to the most widespread type of magnetic fluids, i.e. magnetite particles, in a hydrocarbon medium stabilized by oleic acid.

Acknowledgements. This study was funded by the RFBR (research project No. 6-52-12035/17). I.A.S. is thankful for financial support from the Grant of the President of the Russian Federation for young Russian scientists (contract No. 14.Z56.16.5703-MK). A.O.T. also acknowledges support from the Scholarship of the President of the Russian Federation for young researchers (SP-2683.2015.1).

\section{References}

[1] R.E. Rosensweig. Ferrohydrodynamics (Cambridge Monographs on Mechanics and Applied Mathematics, New-York, 1985).

[2] S. Odenbach. Colloidal Magnetic Fluids: Basics, Development and Application of Ferrofluids (Lecture Notes in Physics, Berlin, Springer, 2009).

[3] S. Chikazumi. Physics of Ferromagnetism (2nd Edition, Oxford University Press, 1997).

[4] V.M. Polunin. Acoustics of Nanodispersed Magnetic Fluids (New York- London, CRC Press, CISP, 2015).

[5] V.M. Polunin, A.M. Storozhenko, P.A. Ryapolov. Mechanics of Liquid Nano- and Microdispersed Magnetic Media (CRC Press Taylor and Francis Group, CISP, Great Britain, 2017).

[6] A.M. Storozhenko, V.M. Polunin. Interaction of physical fields in the acoustomagnetic effect. Acoustical Physics, vol. 58 (2012), no. 2, pp. 180 186.

[7] S.G. Yemelyanov, V.M. Polunin, A.M.Storozhenko et al. Sound speed in a non-uniformly magnetized magnetic fluid. Magnetohydrodynamics, vol. 47 (2011), no. 1, pp. 29-39.

[8] E. Skuchik. Fundamentals of Acoustics (Moscow, Foreign Literature, 1959), vol. 2. 
[9] A.M. Storozhenko, V.M. Polunin, A.O. Tantsyura et al. Study of demagnetizing field induced by a sound wave. Acoustical Physics, vol. 59 (2013), no. 6, pp. 662-666.

[10] D.X. Chen, E. Pardo, A. Sanchez. Fluxmetric and magnetometric demagnetizing factors for cylinders. J. Magn. Magn. Mater., vol. 306 (2006), no. 1, pp. $135-146$.

[11] I.A. Shabanova, A.M. Storozhenko, V.M. Polunin. Determination of sound speed in a magnetic fluid using the acoustomagnetic effect. International Journal of Applied Engineering Research, vol. 11 (2016), no 23, pp. 11171-11175.

[12] S.V. Dyachenko, M.A. Vaseshenkova, K.D. Martinson et al. Synthesis and properties of magnetic fluids produced on the basis of magnetite particles. Russian Journal of Applied Chemistry, vol. 89 (2016), no. 5, pp. 690-696.

[13] T. Sawada, H. Nishiyama, T. Tabata. Influence of a magnetic field on ultrasound propagation in a magnetic fluid. J. Magn. Magn. Mater., vol. 252 (2002), pp. 186-188.

[14] V.M. Polunin, N.S. Kobelev, P.A. Ryapolov et al. On the estimation of physical parameters of magnetic nanoparticles in magnetic fluid. Magnetohydrodynamics, vol. 46 (2010), no. 1, pp. 31-40.

[15] A. Józefrczak, A. Skumiel, M. Labowski. Effects of biocompatible coating of nanoparticles on acoustics property of the magnetic fluid. J. Magn. Magn. Mater., vol. 290-291 (2005), part 1, pp. 265-268.

[16] A.R. Baev, E.V. Korobko, Z.A. Novikova. Acoustical properties of magnetorheological fluids under applied magnetic field. Journal of Intelligent Material Systems and Structures, vol. 26 (2015), no. 14, pp. 1913-1919.

Received 27.12.2017 